\title{
Gene-gene and gene-environment interactions on cord blood total lgE in Chinese Han children
}

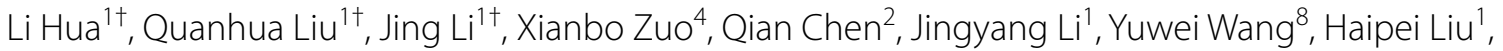
Zhaobo Shen ${ }^{5}$, Yi Li ${ }^{5}$, Zenan Ma ${ }^{6}$, Shengdong Dong ${ }^{7}$, Ruoxu Ji ${ }^{1}$, Dingzhu Fang ${ }^{1}$, Yi Chen ${ }^{1}$, Wenwei Zhong ${ }^{1}$, Jun Zhang ${ }^{2}$, Jianhua Zhang ${ }^{1 *}$ and Yixiao Bao ${ }^{1,3^{*}}$

\begin{abstract}
Background: $I L 13, I L 4, I L 4 R A, F C E R 1 B$ and $A D R B 2$ are susceptible genes of asthma and atopy. Our previous study has found gene-gene interactions on asthma between these genes in Chinese Han children. Whether the interactions begin in fetal stage, and whether these genes interact with prenatal environment to enhance cord blood lgE (CBlgE) levels and then cause subsequent allergic diseases have yet to be determined. This study aimed to determine whether there are gene-gene and gene-environment interactions on CBlgE elevation among the aforementioned five genes and prenatal environmental factors in Chinese Han population.
\end{abstract}

Methods: 989 cord blood samples from a Chinese birth cohort were genotyped for nine single-nucleotide polymorphisms (SNPs) in the five genes, and measured for CBlgE levels. Prenatal environmental factors were collected using a questionnaire. Gene-gene and gene-environment interactions were analyzed with generalized multifactor dimensionality methods.

Results: A four-way gene-gene interaction model (IL13 rs20541, IL 13 rs 1800925, IL4 rs2243250 and ADRB2 rs 1042713) was regarded as the optimal one for $\mathrm{CBlgE}$ elevation (testing balanced accuracy $=0.5805, P=9.03 \times 10^{-4}$ ). Among the four SNPS, only IL 13 rs 20541 was identified to have an independent effect on elevated CBlgE (odds ratio $(O R)=1.36$, $P=3.57 \times 10^{-3}$ ), while the other three had small but synergistic effects. Carriers of IL13 rs 20541 TT, IL 13 rs 1800925 CT/TT, IL4 rs2243250 TT and ADRB2 rs 1042713 AA were estimated to be at more than fourfold higher risk for CBlgE elevation $\left(\mathrm{OR}=4.14, P=2.69 \times 10^{-2}\right)$. Gene-environment interaction on elevated $\mathrm{CBlgE}$ was found between IL4 rs2243250 and maternal atopy $\left(\mathrm{OR}=1.41, P=2.65 \times 10^{-2}\right)$.

Conclusions: Gene-gene interaction between IL13 rs20541, IL 13 rs1800925, IL4 rs2243250 and ADRB2 rs1042713, and gene-environment interaction between IL4 rs2243250 and maternal atopy begin in prenatal stage to augment IgE production in Chinese Han children.

Keywords: Cord blood, IgE, Gene-gene interaction, Gene-environment interaction

*Correspondence: zjh12195@126.com; drbaoyixiao@163.com †Li Hua, Quanhua Liu and Jing Li contributed equally to the article ${ }^{1}$ Department of Pediatric Pulmonology, Xin Hua Hospital, Shanghai Jiao Tong University School of Medicine, 1665 Kongjiang Road, Shanghai 200092, China

Full list of author information is available at the end of the article

\section{Introduction}

The worldwide prevalence of allergic diseases has dramatically increased during the past few decades, resulting in heavy burden to the whole society and huge medical expenditure around the world [1]. Allergic diseases have long been attributed to IgE-mediated

c) The Author(s) 2021. This article is licensed under a Creative Commons Attribution 4.0 International License, which permits use, sharing, adaptation, distribution and reproduction in any medium or format, as long as you give appropriate credit to the original author(s) and the source, provide a link to the Creative Commons licence, and indicate if changes were made. The images or other third party material in this article are included in the article's Creative Commons licence, unless indicated otherwise in a credit line to the material. If material is not included in the article's Creative Commons licence and your intended use is not permitted by statutory regulation or exceeds the permitted use, you will need to obtain permission directly from the copyright holder. To view a copy of this licence, visit http://creativecommons.org/licenses/by/4.0/. The Creative Commons Public Domain Dedication waiver (http://creativecommons.org/ publicdomain/zero/1.0/) applies to the data made available in this article, unless otherwise stated in a credit line to the data. 
inflammatory responses [2]. Evidence has demonstrated that regulation of IgE production may begin in utero, reflected in the levels of cord blood IgE (CBIgE) [3]. Elevated CBIgE has been shown to be a risk factor for the subsequent development of allergic diseases [4]. Recent studies have indicated that certain genes and environmental factors may interact to elevate CBIgE levels [5-7], with the heritability estimated around $84-95 \%[8]$.

Ober and Hoffjan reviewed 118 genes associated with asthma or atopy, among which 25 have been replicated in six or more independent samples and thus are considered to be true susceptibility genes [9]. The elite group of susceptible genes of asthma and atopy replicated in more than ten different studies include IL13, IL4, IL4RA, $F C E R 1 B$ and $A D R B 2$, five important inflammatory genes associated with IgE levels [10-12]. Our previous study has found that gene-gene interactions on childhood asthma exist between these genes in Chinese Han population [13]. Whether the gene-gene interactions among the aforementioned five genes begin in fetal stage, and whether these genes interact with prenatal environment to enhance CBIgE production and then cause subsequent allergic diseases have yet to be determined.

This study attempts to explore whether there are gene-gene and gene-environment interactions on CBIgE elevation among genetic variants in IL13, IL4, ILARA, FCER $1 B$ and $A D R B 2$ genes and prenatal environmental factors in Chinese Han population. This is the first study to investigate gene-gene and gene-environment interactions on CBIgE in the mainland of China. Elucidation of genetic and environmental determinants of CBIgE may allow for detection and prevention of allergic sensitization in early life.

\section{Methods}

\section{Study participants}

This study included 989 Chinese Han children from the Shanghai Allergy Cohort, which was a prospective birth cohort with infants recruited between 2012 and 2013 at two large tertiary hospitals in Shanghai, Xinhua Hospital and the International Peace Maternity \& Child Health Hospital. Written informed consent was obtained from the mothers prior to delivery. Prenatal and perinatal epidemiologic and clinical information along with cord blood samples were collected by trained research nurses. The study was approved by the Ethics Committee of Xinhua Hospital and the International Peace Maternity \& Child Health Hospital (approval number: XHEC-C-2012-003), and conducted according to the principles in the Declaration of Helsinki.

\section{Epidemiologic and clinical information collection}

Trained research nurses conducted face-to-face interviews using structured questionnaires, collecting information on maternal age, height, prepregnancy weight, education level, maternal atopy, prenatal pet exposure, prenatal active or secondhand smoking, and family income. Maternal atopy was referred to those mothers who had asthma, allergic rhinitis or atopic dermatitis along with detectable specific IgE. Prenatal pet exposure was defined as keeping cats or dogs at home during pregnancy.

Information on parity, previous pregnancy, gestational age, date of birth, delivery mode, infants' gender, birth weight and antenatal complications was obtained from medical records.

\section{CBIgE measurement}

CBIgE levels were determined by using ImmunoCAP Total IgE Low Range Assay [5] on the Phadia 250 (Thermo Scientific ${ }^{\mathrm{TM}}$, Waltham, Massachusetts, USA) according to the standard manufacturer's protocols. Elevation of CBIgE levels was cut-off at $\geq 0.5 \mathrm{KU} / \mathrm{L}$ as previously described $[5,6]$.

\section{Selection of genes and single nucleotide polymorphisms}

This study focused on five candidate genes, including IL13, IL4, IL4RA, FCER1B and ADRB2, which are key inflammatory genes affecting IgE levels [10-12] and had been found associated with asthma or atopy by more than ten different studies [9]. Our previous study had identified gene-gene interactions on asthma between these genes in Chinese Han children [13]. Within these genes, nine known functional single-nucleotide polymorphisms (SNPs) [13] with minor allele frequency greater than $10 \%$ were chosen for analysis, as shown in Table 1.

Table 1 Candidate genes and SNPs analyzed in this study

\begin{tabular}{lllll}
\hline Gene & SNP & rs Number & Chromosome position & Location \\
\hline IL13 & $-1112 C>T$ & rs 1800925 & $5: 132,657,117$ & Promoter \\
IL13 & $+1923 C>T$ & rs1295686 & $5: 132,660,151$ & Intron 3 \\
IL13 & R110Q & rs20541 & $5: 132,660,272$ & Exon 4 \\
IL4 & $-590 C>T$ & rs2243250 & $5: 132,673,462$ & Promoter \\
ADRB2 & R16G & rs 1042713 & $5: 148,826,877$ & Exon1 \\
FCER1B & $-109 C>T$ & rs1441586 & $11: 60,088,555$ & Promoter \\
FCER1B & E237G & rs569108 & $11: 60,095,631$ & Exon7 \\
IL4RA & I75V & rs1805010 & $16: 27,344,882$ & Exon 5 \\
IL4RA & Q551R & rs1801275 & $16: 27,363,079$ & Exon 12 \\
\hline
\end{tabular}

SNP single-nucleotide polymorphism, $r$ reference SNP 


\section{Genotyping}

Genomic DNA was extracted from cord blood using QIAamp DNA Blood Mini Kit (QIAGEN, Hilden, Germany). Genotyping of the nine SNPs was performed by matrix-assisted laser desorption / ionization time of flight mass spectrometry (MALDI-TOF MS) [14] using the MassARRAY iPLEX platform (Sequenom Inc, San Diego, CA, USA) according to the manufacturer's instructions. Laboratory personnel were blinded to CBIgE status. The overall call rate was 98.6\%. Genotyping quality control included 5\% duplicate and negative samples. Genotyping concordance rate was higher than $98 \%$.

\section{Statistical analysis}

Associations between CBIgE elevation and the epidemiologic characteristics of the study subjects were assessed by the $\chi^{2}$ test. The Hardy-Weinberg equilibrium test for each of the nine SNPs was performed in the total population with the $\chi^{2}$ statistics. Association of elevated CBIgE in subjects with each SNP was analyzed by using the Pearson's $X^{2}$ test. In addition to the allelic test of association, dominant and recessive genetic models were tested for the nine SNPs by logistic regression analysis. $P$ value, odds ratio (OR) and 95\% confidence interval ( $95 \% \mathrm{CI})$ were calculated by using the PLINK program (http://pngu.mgh.harvard.edu/ purcell/plink/). A twotailed $P$ value $\leq 0.0055$ after Bonferroni Multiple Testing correction was considered statistically significant.

Gene-gene interactions were analyzed with GMDR (Version 1.0), which is a free, open-source interaction analysis tool, aimed to perform gene-gene interaction with generalized multifactor dimensionality reduction (GMDR) methods [15]. The model that maximizes the testing balanced accuracy (TBA) and minimizes the statistical significance is selected. TBA indicates the accuracy of classification of cases and controls. Heuristically, a satisfactory TBA is higher than 0.55. Gene-gene interactions revealed by GMDR analyses were validated by $\chi^{2}$ tests. Gene-environment interactions were evaluated by logistic regression analysis and GMDR approach. Linkage disequilibrium (LD) was calculated for the SNPs located on one chromosome. The detection power of the sample size in this study was 0.88 based on the minor allele frequency of 0.25 and its OR for CBIgE elevation at 1.30 .

\section{Results}

\section{Association between CBIgE elevation} and the epidemiologic characteristics of the study subjects There were 989 Chinese Han infants in this study, of whom $27.1 \%$ had elevated CBIgE levels. Table 2
Table 2 Associations between CBlgE elevation and the epidemiologic characteristics of the study subjects

\begin{tabular}{|c|c|c|c|}
\hline Phenotypes & N (\%) & $\begin{array}{l}\text { Elevated rate } \\
\text { of CBIgE }\end{array}$ & $P$ value ${ }^{*}$ \\
\hline \multicolumn{4}{|l|}{ Maternal age (y) } \\
\hline$<25$ & $62(6.4)$ & $17(27.4)$ & \multirow[t]{4}{*}{$6.92 \times 10^{-1}$} \\
\hline $25-29$ & $500(51.9)$ & $128(25.6)$ & \\
\hline $30-34$ & $325(33.7)$ & $92(28.3)$ & \\
\hline$\geq 35$ & $77(8.0)$ & $24(31.2)$ & \\
\hline \multicolumn{4}{|c|}{ Maternal prepregnancy BMI $\left(\mathrm{kg} / \mathrm{m}^{2}\right)$} \\
\hline$<18.5$ & $150(15.6)$ & $42(28.0)$ & \multirow[t]{4}{*}{$5.79 \times 10^{-1}$} \\
\hline $18.5-24.9$ & $694(72.1)$ & $182(26.2)$ & \\
\hline $25-29.9$ & $92(9.6)$ & $29(31.5)$ & \\
\hline$\geq 30$ & $26(2.7)$ & $9(34.6)$ & \\
\hline \multicolumn{4}{|l|}{ Maternal education } \\
\hline Middle school or lower & $28(2.9)$ & $6(21.4)$ & \multirow[t]{3}{*}{$5.48 \times 10^{-}$} \\
\hline High school & $112(11.6)$ & $27(24.1)$ & \\
\hline College or higher & $825(85.5)$ & $230(27.9)$ & \\
\hline \multicolumn{4}{|l|}{ Family income (CNY) } \\
\hline$<100 \mathrm{~K}$ & $273(27.6)$ & $74(27.1)$ & \multirow[t]{3}{*}{$9.98 \times 10^{-1}$} \\
\hline$\geq 100 \mathrm{~K}$ & $540(54.6)$ & $146(27.0)$ & \\
\hline Unknown & $176(17.8)$ & $48(27.3)$ & \\
\hline \multicolumn{4}{|l|}{ Maternal atopy ${ }^{\mathrm{a}}$} \\
\hline No & $824(86.5)$ & $219(26.6)$ & \multirow[t]{2}{*}{$2.17 \times 10^{-1}$} \\
\hline Yes & $129(13.5)$ & $41(31.8)$ & \\
\hline \multicolumn{4}{|l|}{ Prenatal pet exposure ${ }^{b}$} \\
\hline No & $857(89.1)$ & $234(27.3)$ & \multirow[t]{2}{*}{$8.90 \times 10^{-1}$} \\
\hline Yes & $105(10.9)$ & $28(26.7)$ & \\
\hline \multicolumn{4}{|c|}{ Prenatal active or secondhand smoking } \\
\hline No & $577(59.9)$ & $158(27.4)$ & \multirow[t]{2}{*}{$9.32 \times 10^{-1}$} \\
\hline Yes & $387(40.1)$ & $105(27.1)$ & \\
\hline \multicolumn{4}{|l|}{ Parity } \\
\hline None & $868(89.9)$ & $235(27.1)$ & \multirow[t]{2}{*}{$9.20 \times 10^{-1}$} \\
\hline$\geq 1$ & $98(10.1)$ & $27(27.6)$ & \\
\hline \multicolumn{4}{|l|}{ Previous pregnancy } \\
\hline None & $629(65.1)$ & $169(26.9)$ & \multirow[t]{2}{*}{$8.08 \times 10^{-1}$} \\
\hline$\geq 1$ & $337(34.9)$ & $93(27.6)$ & \\
\hline \multicolumn{4}{|l|}{ Gestational age (wk) } \\
\hline$<37$ & $33(3.4)$ & $7(21.2)$ & \\
\hline $37-39$ & $686(71.0)$ & $196(28.6)$ & \multirow[t]{2}{*}{$2.70 \times 10^{-1}$} \\
\hline$\geq 40$ & $247(25.6)$ & $59(23.9)$ & \\
\hline \multicolumn{4}{|l|}{ Season of birth } \\
\hline Summer (Jun.-Aug.) & $170(17.7)$ & $46(27.1)$ & \multirow[t]{3}{*}{$4.90 \times 10^{-1}$} \\
\hline Autumn (Sep.-Nov.) & $451(47.1)$ & $131(29.0)$ & \\
\hline Winter (Dec.-Feb.) & $337(35.2)$ & $85(25.2)$ & \\
\hline \multicolumn{4}{|l|}{ Delivery mode } \\
\hline Vaginal & $229(23.7)$ & $50(21.8)$ & $3.93 \times 10^{-2}$ \\
\hline Cesarean section & $737(76.3)$ & $212(28.8)$ & \\
\hline Gender & & & \\
\hline Boy & $499(51.7)$ & $156(31.3)$ & $2.28 \times 10^{-3}$ \\
\hline Girl & $466(48.3)$ & $105(22.5)$ & \\
\hline
\end{tabular}


Table 2 (continued)

\begin{tabular}{lcrl}
\hline Phenotypes & N (\%) & $\begin{array}{l}\text { Elevated rate } \\
\text { of CBIgE }\end{array}$ & $\boldsymbol{P}^{\text {value }}$ \\
\hline $\begin{array}{l}\text { Birth weight (g) } \\
<2500\end{array}$ & $24(2.5)$ & $8(33.3)$ & $7.86 \times 10^{-1}$ \\
$2500-4000$ & $857(88.7)$ & $231(27.0)$ & \\
$\geq 4000$ & $85(8.8)$ & $23(27.1)$ & \\
Antenatal complications ${ }^{c}$ & & & \\
No & $763(81.2)$ & $206(27.0)$ & $6.26 \times 10^{-1}$ \\
Yes & $177(18.8)$ & $51(28.8)$ &
\end{tabular}

The missing data: maternal age $(n=25)$; maternal prepregnancy BMI $(n=27)$; maternal education $(n=24)$; maternal atopy $(n=36)$; prenatal pet exposure $(n=27)$; prenatal active or secondhand smoking $(n=25)$; parity $(n=23)$; previous pregnancy $(n=23)$; gestational age $(n=23)$; season of birth $(n=31)$; delivery mode $(n=23)$; gender $(n=24)$; birth weight $(n=23)$; antenatal complications $(n=49)$. The missing data were not from the same individuals for each variable

CBIgE cord blood IgE

${ }^{a}$ Maternal atopy was referred to those mothers who had asthma, allergic rhinitis or atopic dermatitis along with detectable specific $\lg \mathrm{E}$

${ }^{\mathrm{b}}$ Keeping cats or dogs at home during pregnancy

' Pregnancy hypertension, diabetes, infection or intrauterine growth retardation ${ }^{*} \mathrm{X}^{2}$ test was used to analyze associations between CBlgE elevation and the epidemiologic characteristics

presents the distribution of CBIgE concentrations by epidemiologic characteristics of the study subjects. Cesarean section and male gender were associated with elevated CBIgE levels $(P<0.05)$.

\section{Association between CBIgE elevation and single SNPs}

All the nine SNPs were in Hardy-Weinberg equilibrium $(\mathrm{P}>0.05)$. As shown in Table 3, SNPs IL13 rs1295686 and IL13 rs20541 were solely associated with CBIgE elevation. The A allele of rs1295686 (OR=1.37, $\left.P=2.73 \times 10^{-3}\right)$ and T allele of rs20541 $\left(\mathrm{OR}=1.36, P=3.57 \times 10^{-3}\right)$ were significantly increased in elevated CBIgE group compared with normal group. The most significant association with CBIgE elevation was found under recessive model for the two SNPs. Significant association with CBIgE elevation was not found among the other seven loci $(\mathrm{P}>0.0055$, after Bonferroni Multiple Testing correction).

\section{Gene-gene interactions on CBIgE elevation}

Gene-gene interactions on CBIgE elevation were explored among all the nine SNPs by GMDR approach. Totally, there were four models exhibiting a TBA higher than 0.55, as shown in Table 4. Based on the TBA and $P$ values, significant multi-loci interactions were found in the four models $(P<0.05)$. Among them, the fourway interaction model (IL13 rs20541, IL13 rs1800925, IL4 rs2243250 and ADRB2 rs1042713) which showed the highest TBA and lowest $P$ value (TBA $=0.5805$, $P=9.03 \times 10^{-4}$ ), was regarded as the optimal one. As the four SNPs that made up the optimal model are located on one chromosome, pairwise LD of them was calculated $\left(r^{2}<0.3\right)$, indicating a low LD between them. Interactions between the four SNPs of the optimal model were further validated by $X^{2}$ tests. Table 5 shows that individuals carrying IL13 rs20541 TT, IL13 rs1800925 CT/TT, IL4 rs2243250 TT and ADRB2 rs1042713 AA had a significantly higher risk of CBIgE elevation compared with those without any of the four risk genotypes $\left(\mathrm{OR}=4.14, P=2.69 \times 10^{-2}\right)$, and also greater than those with less than four risk genotypes.

\section{Gene-environment interactions on CBIgE elevation}

Logistic regression analysis and GMDR approach were applied to search the potential gene-environment interactions on CBIgE elevation between the nine SNPs and environmental factors including prenatal pet exposure, prenatal active or secondhand smoking, maternal atopy, maternal age, maternal prepregnancy BMI, delivery mode, infants' gender and season of birth. By using logistic regression analysis, it was found that $\mathrm{C}$ allele of IL4 rs2243250 interacted with maternal atopy to elevate $\mathrm{CBIgE}$ levels $\left(\mathrm{OR}=1.41, P=2.65 \times 10^{-2}\right)$, as shown in Table 6. However, no significant geneenvironment interaction was found by GMDR analysis.

\section{Discussion}

IgE-mediated reaction is the central component of allergic diseases. Five key inflammatory genes affecting IgE levels, including IL13, IL4, ILARA, FCER1B and $A D R B 2$ [10-12], have been demonstrated associated with asthma or atopy by more than ten different studies [9]. Our previous study has found that gene-gene interactions on asthma exist between these genes in Chinese Han children [13]. This study attempted to determine whether the interactions begin in utero, and whether these genes interact with prenatal environmental factors to increase CBIgE levels and induce subsequent allergic diseases. Of the models tested using GMDR approach, the fourway gene-gene interaction model consisting of IL13 rs20541, IL13 rs1800925, IL4 rs2243250 and ADRB2 rs1042713 was chosen as the optimal one for CBIgE elevation based on its TBA and $P$ value. Among the four SNPs, only IL13 rs20541 was identified to have an independent effect on CBIgE elevation, while the other three had small but synergistic effects. Carriers of IL13 rs20541 TT, IL13 rs1800925 CT/TT, IL4 rs2243250 TT and $A D R B 2$ rs1042713 AA were estimated to be at more than fourfold higher risk for CBIgE elevation. Among these genes and prenatal environmental factors, only IL4 rs2243250 and maternal atopy were found to have interactions on elevated CBIgE. This is the first study 
Table 3 Genetic effects of single SNPs on CBlgE elevation

\begin{tabular}{|c|c|c|c|c|c|c|c|}
\hline SNP & CBIgE levels & Risk allele & $\begin{array}{l}\text { Risk allele } \\
\text { frequency, } \\
\mathrm{n}(\%)\end{array}$ & $P$ value ${ }^{*} \mathrm{OR}(95 \% \mathrm{Cl})$ & $\begin{array}{l}\text { Genotype frequency } A A / A B / B B \\
n(\%)\end{array}$ & $\begin{array}{l}\text { Dominant }^{\dagger} \\
P \text { value }^{\ddagger} \\
\text { OR(95\%Cl) }\end{array}$ & $\begin{array}{l}\text { Recessive }^{\dagger} \\
P \text { value }^{\ddagger} \\
\text { OR(95\%Cl) }\end{array}$ \\
\hline 1413 & & & & & $\mathrm{CC} / \mathrm{CT} / \mathrm{TT}$ & & \\
\hline \multirow[t]{2}{*}{ rs1800925 } & Elevated & T & $102(19.2)$ & $5.17 \times 10^{-2}$ & $170(63.9) / 90(33.8) / 6(2.3)$ & $2.79 \times 10^{-2}$ & $9.77 \times 10^{-1}$ \\
\hline & Normal & & $223(15.5)$ & $1.29(1.00-1.67)$ & $512(71.2) / 191(26.6) / 16(2.2)$ & $1.40(1.04-1.88)$ & $1.01(0.39-2.62)$ \\
\hline IL13 & & & & & GG/GA/AA & & \\
\hline \multirow[t]{2}{*}{ rs1295686 } & Elevated & A & $204(38.5)$ & $2.73 \times 10^{-3}$ & $97(36.6) / 132(49.8) / 36(13.6)$ & $8.71 \times 10^{-3}$ & $2.18 \times 10^{-2}$ \\
\hline & Normal & & $451(31.3)$ & $1.37(1.12-1.69)$ & $331(46.0) / 327(45.4) / 62(8.6)$ & $1.47(1.10-1.97)$ & $1.67(1.08-2.58)$ \\
\hline IL13 & & & & & $\mathrm{CC} / \mathrm{CT} / \mathrm{TT}$ & & \\
\hline \multirow[t]{2}{*}{ rs20541 } & Elevated & $\mathrm{T}$ & $203(38.5)$ & $3.57 \times 10^{-3}$ & $97(36.7) / 131(49.6) / 36(13.6)$ & $1.23 \times 10^{-2}$ & $2.07 \times 10^{-2}$ \\
\hline & Normal & & $453(31.5)$ & $1.36(1.11-1.68)$ & $329(45.7) / 329(45.7) / 62(8.6)$ & $1.45(1.08-1.94)$ & $1.68(1.08-2.60)$ \\
\hline IL4 & & & & & $\mathrm{TT} / \mathrm{TC} / \mathrm{CC}$ & & \\
\hline \multirow[t]{2}{*}{ rs2243250 } & Elevated & $C$ & $109(20.6)$ & $7.55 \times 10^{-1}$ & $168(63.4) / 85(32.1) / 12(4.5)$ & $7.00 \times 10^{-1}$ & $9.71 \times 10^{-1}$ \\
\hline & Normal & & $287(19.9)$ & $1.04(0.81-1.33)$ & $466(64.7) / 221(30.7) / 33(4.6)$ & $1.06(0.79-1.42)$ & $0.99(0.50-1.94)$ \\
\hline ADRB2 & & & & & $\mathrm{AA} / \mathrm{AG} / \mathrm{GG}$ & & \\
\hline \multirow[t]{2}{*}{ rs1042713 } & Elevated & A & $296(61.7)$ & $1.41 \times 10^{-1}$ & $97(40.4) / 102(42.5) / 41(17.1)$ & $8.81 \times 10^{-2}$ & $5.94 \times 10^{-1}$ \\
\hline & Normal & & $763(57.8)$ & $1.17(0.95-1.46)$ & $226(34.2) / 311(47.1) / 123(18.6)$ & $0.77(0.57-1.04)$ & $0.90(0.61-1.33)$ \\
\hline FCER1B & & & & & $\mathrm{TT} / \mathrm{TC} / \mathrm{CC}$ & & \\
\hline \multirow[t]{2}{*}{ rs1441586 } & Elevated & C & $187(35.4)$ & $3.41 \times 10^{-1}$ & $108(40.9) / 125(47.3) / 31(11.7)$ & $3.82 \times 10^{-1}$ & $5.10 \times 10^{-1}$ \\
\hline & Normal & & $477(33.1)$ & $1.11(0.90-1.37)$ & $317(44.0) / 329(45.7) / 74(10.3)$ & $1.14(0.85-1.51)$ & $1.16(0.74-1.81)$ \\
\hline FCER1B & & & & & $\mathrm{TT} / \mathrm{TC} / \mathrm{CC}$ & & \\
\hline \multirow[t]{2}{*}{ rs569108 } & Elevated & $C$ & $91(17.0)$ & $7.26 \times 10^{-1}$ & $184(68.7) / 77(28.7) / 7(2.6)$ & $7.47 \times 10^{-1}$ & $8.20 \times 10^{-1}$ \\
\hline & Normal & & $235(16.3)$ & $1.05(0.80-1.37)$ & $502(69.7) / 201(27.9) / 17(2.4)$ & $1.05(0.78-1.42)$ & $1.11(0.45-2.71)$ \\
\hline IL $4 R A$ & & & & & GG/GA/AA & & \\
\hline \multirow[t]{2}{*}{ rs1805010 } & Elevated & G & $274(51.9)$ & $3.22 \times 10^{-1}$ & $72(27.3) / 130(49.2) / 62(23.5)$ & $3.84 \times 10^{-1}$ & $4.59 \times 10^{-1}$ \\
\hline & Normal & & $708(49.4)$ & $1.11(0.91-1.35)$ & $176(24.5) / 356(49.7) / 185(25.8)$ & $0.87(0.63-1.19)$ & $0.88(0.63-1.23)$ \\
\hline IL $4 R A$ & & & & & $\mathrm{AA} / \mathrm{AG} / \mathrm{GG}$ & & \\
\hline \multirow[t]{2}{*}{ rs1801275 } & Elevated & A & $442(83.4)$ & $6.66 \times 10^{-1}$ & $178(67.2) / 86(32.5) / 1(0.4)$ & $6.97 \times 10^{-1}$ & $3.09 \times 10^{-2}$ \\
\hline & Normal & & 1189 (82.6) & $1.06(0.81-1.38)$ & 493(68.5)/203(28.2)/24(3.3) & $1.06(0.79-1.43)$ & $0.11(0.01-0.82)$ \\
\hline
\end{tabular}

$S N P$ single-nucleotide polymorphism, $C B I g E$ cord blood IgE, $O R$ odds ratio, $C I$ confidence interval

${ }^{*} P$ Values for Pearson's $X^{2}$ tests

${ }^{\dagger}$ Dominant model $(A A$ vs $A B+B B)$ and recessive model $(A A+A B$ vs $B B)$, where $A$ is the major allele and $B$ is the minor allele

${ }^{\ddagger} P$ Values for logistic analyses

Table 4 Summary of gene-gene interactions for CBlgE elevation by GMDR analysis

\begin{tabular}{|c|c|c|}
\hline Interacting SNPs & TBA & $P$ value \\
\hline IL13 rs20541 × ADRB2 rs1042713 & 0.5621 & $1.07 \times 10^{-2}$ \\
\hline IL 13 rs $1800925 \times$ IL 4 rs $2243250 \times$ ADRB2 rs 1042713 & 0.5591 & $7.77 \times 10^{-3}$ \\
\hline IL 13 rs $20541 \times$ IL 13 rs $1800925 \times$ IL4 rs $2243250 \times$ ADRB2 rs 1042713 & 0.5805 & $9.03 \times 10^{-4}$ \\
\hline IL 13 rs $20541 \times$ IL 13 rs $1800925 \times$ IL 13 rs $1295686 \times$ IL4 rs $2243250 \times A D R B 2$ rs 1042713 & 0.5724 & $1.53 \times 10^{-3}$ \\
\hline
\end{tabular}

CBIgE cord blood IgE, GMDR generalized multifactor dimensionality reduction, SNP single-nucleotide polymorphism, TBA Testing balanced accuracy

to elucidate genetic and environmental determinants of CBIgE in Han population of mainland China.

To our knowledge, this study is also the first to identify gene-gene interactions between IL13 rs20541, IL13 rs1800925, IL4 rs2243250 and ADRB2 rs1042713 on
CBIgE elevation. IL13 and IL4 genes encode cytokines interleukin 13 (IL13) and IL4, which share a common signaling pathway in binding to their receptors on human $\mathrm{B}$ cells, and switch immunoglobulin production from IgM to IgE [16]. ADRB2 gene encodes Beta2-adrenergic 
Table 5 Interactions between IL 13 rs20541, IL 13 rs1800925, IL4 rs2243250 and ADRB2 rs1042713 genotypes for CBlgE elevation

\begin{tabular}{|c|c|c|c|c|}
\hline \multirow{2}{*}{$\begin{array}{l}\text { Number of risk genotype for the } \\
\text { four SNPs }{ }^{a}\end{array}$} & \multicolumn{2}{|l|}{ CBIgE levels } & \multirow[t]{2}{*}{$P$ value ${ }^{\dagger}$} & \multirow[t]{2}{*}{ OR $(95 \% \mathrm{Cl})$} \\
\hline & Elevated, n (\%) & Normal, n (\%) & & \\
\hline 0 & $31(13.2)$ & $110(16.8)$ & & 1 \\
\hline 1 & $92(39.1)$ & $265(40.4)$ & $3.78 \times 10^{-1}$ & $1.23(0.78-1.96)$ \\
\hline 2 & $71(30.2)$ & $217(33.1)$ & $5.42 \times 10^{-1}$ & $1.16(0.72-1.88)$ \\
\hline 3 & $34(14.5)$ & $58(8.8)$ & $1.28 \times 10^{-2}$ & $2.08(1.16-3.72)$ \\
\hline 4 & $7(3.0)$ & $6(0.9)$ & $2.69 \times 10^{-2}$ & $4.14(1.30-13.22)$ \\
\hline
\end{tabular}

CBIgE cord blood IgE, SNP single-nucleotide polymorphism, OR odds ratio, $C l$ confidence interval

${ }^{a}$ Risk genotypes were TT, CT/TT, TT, and AA for rs20541, rs1800925, rs2243250, and rs1042713, respectively

${ }^{+} P$ Values for $X^{2}$ tests

Table 6 Interactions between the nine SNPs and maternal atopy for CBlgE elevation

\begin{tabular}{lllll}
\hline Gene & SNP & Minor allele & OR(95\%Cl) & Pvalue $^{*}$ \\
\hline IL13 & rs1800925 & T & $1.21(0.86-1.71)$ & $2.81 \times 10^{-1}$ \\
IL13 & rs1295686 & A & $0.89(0.67-1.20)$ & $4.53 \times 10^{-1}$ \\
IL13 & rs20541 & T & $0.91(0.68-1.22)$ & $5.25 \times 10^{-1}$ \\
IL4 & rs2243250 & C & $1.41(1.04-1.91)$ & $2.65 \times 10^{-2}$ \\
ADRB2 & rs1042713 & G & $0.86(0.66-1.14)$ & $2.94 \times 10^{-1}$ \\
FCER1B & rs1441586 & C & $1.21(0.91-1.59)$ & $1.91 \times 10^{-1}$ \\
FCER1B & rs569108 & C & $1.19(0.84-1.69)$ & $3.19 \times 10^{-1}$ \\
IL4RA & rs1805010 & A & $0.89(0.68-1.16)$ & $3.77 \times 10^{-1}$ \\
IL4RA & rs1801275 & G & $1.23(0.88-1.72)$ & $2.31 \times 10^{-1}$ \\
\hline
\end{tabular}

SNP single-nucleotide polymorphism, CBIgE cord blood IgE, OR odds ratio, $\mathrm{Cl}$ confidence interval

${ }^{*} P$ Values were tested by multivariate logistic regression, adjusted for other genes, but not for other environmental factors

receptor (ADRB2). Stimulation of ADRB2 on B cells responding to allergen enhances $\operatorname{IgE}$ production via a unique signaling pathway, independently of class switch recombination $[17,18]$. IL13, IL4 and ADRB2 are all associated with IgE levels. IL13 rs20541 TT genotype, IL13 rs1800925 T allele, IL4 rs2243250 TT genotype and $A D R B 2$ rs1042713 AA genotype have been associated with increased IL13 concentration [19], enhanced IL13 promoter activity [20], augmented IL4 levels [21], and decreased downregulation of ADRB2 [22], respectively. How these four varients interact with each other biologically to promote IgE production in prenatal stage need further functional studies in vitro and in vivo.

In this study, gene-environment interaction on elevated CBIgE was found between IL4 rs2243250 and maternal atopy. Maternal atopy has been reported to modify cord blood immune response and it may provide an intrauterine environment that influences fetal immune development and results in allergic predisposition [23-25]. IL4 gene polymorphism affects cytokine IL4 levels [26]. How maternal atopy interacts with IL4 gene variants to enhance antenatal IgE production need future biological studies.

Our study confirmed the independent role of IL13 rs20541 and rs1295686 on CBIgE elevation, and also found the association of cesarean section and male gender with elevated CBIgE levels, consistent with previous reports [3, 5-7, 27]. However, no interactions were identified among them. To date, only a few studies have explored gene-gene and gene-environment interactions on CBIgE elevation. One study in a predominantly black sample reported that three IL13 SNPs (rs1295686, rs1800925 and rs206974) could jointly influence CBIgE concentration [3]. One study in a birth cohort in Korea identified interactions between reactive oxygen species genes, prenatal exposure to home renovation and maternal atopy on CBIgE response [28]. Another study, in a Chinese population in Taiwan, found that IL13 rs20541, male sex and prenatal environmental tobacco smoke interacted on antenatal IgE production [5]. In this study, we found a four-way genetic interactions among IL13 rs20541, IL13 rs1800925, IL4 rs2243250 and ADRB2 rs1042713, and a two-way gene-environment interactions between IL4 rs2243250 and maternal atopy on CBIgE elevation. The variation of the gene-gene and geneenvironment interactions on fetal IgE production may be in part explained by different populations and different genetic and environmental factors focused in different studies. Therefore, when we move forward to identify constellations of interacting genes and environments that influence antenatal IgE production, replication studies in different populations are required.

There are some limitations in this study. First, only five genes (IL13, IL4, IL4RA, FCER1B and ADRB2) were chosen as candidate genes. However, these five genes are susceptible genes of asthma and atopy replicated in more than ten different studies [9], and our previous study has found that gene-gene interactions on asthma exist between these genes in Chinese Han 
children [13]. Second, the subjects' environmental exposures were evaluated using a self-reported questionnaire, which might lead to an underestimation of the associations of certain environmental exposures. Genes and environmental factors interact to elevate CBIgE concentrations [5-7], with the heritability estimated around 84-95\% [8]. In our future studies, more candidate genes especially those from genomewide association studies should be included and direct measurement of certain environmental exposures is needed. Third, cord blood IgA concentrations were not measured to exclude subjects whose circulation was contaminated by maternal blood. However, previous studies using cord blood IgA levels as an indicator of maternal contamination have reported a very low rate of contamination [29]. Therefore CBIgE is unlikely to be contaminated by maternal IgE [3].

In summary, Gene-gene interaction between IL13 rs20541, IL13 rs1800925, IL4 rs2243250 and ADRB2 rs1042713, and gene-environment interaction between IL4 rs2243250 and maternal atopy begin in fetal stage to increase IgE production in Chinese Han children. After future functional and replication studies, these findings may be translated into specific strategies for early prediction and prevention of allergy.

\section{Acknowledgements}

We heartily thank all the study participants and their parents.

\begin{abstract}
Authors' contributions
$Y X B, L H, J H Z$ and $J Z$ conceived and designed the study. QC and QHL collected and processed the specimens. QHL, LH, JL, ZNM and SDD did the measurement of the specimens. $L H, Q H L, Q C$ and JYL sorted out the questionnaire data. XBZ, LH and YWW conducted the statistical analysis. LH drafted the manuscript. YXB, JHZ, ZBS, YL, WWZ, HPL, RXJ, DZF and YC reviewed and edited the manuscript. All authors read and approved the manuscript.
\end{abstract}

\section{Funding}

This study was funded by the Youth Program of National Nature Science Foundation of China (No. 81900018), the Project for Innovation Teams in Local High Level Universities in Shanghai, the Youth Project of Shanghai Municipal Health Commission (No. 20164Y0136), and the State Key Program of National Nature Science Foundation of China (No. 81530086).

\section{Availability of data and materials}

The data that support the findings of this study are not publicly available due to ethical concerns, but are available from the corresponding authors upon reasonable request.

\section{Declarations}

\section{Ethics approval and consent to participate}

Written informed consent was obtained from the subjects' mothers. The study was approved by the Ethics Committee of Xinhua Hospital and the International Peace Maternity \& Child Health Hospital (approval number: XHEC-C-2012-003), and conducted according to the principles in the Declaration of Helsinki.

\section{Consent for publication}

Not applicable.

\section{Competing interests}

The authors declare that they have no competing interests.

\section{Author details}

${ }^{1}$ Department of Pediatric Pulmonology, Xin Hua Hospital, Shanghai Jiao Tong University School of Medicine, 1665 Kongjiang Road, Shanghai 200092, China. ${ }^{2}$ Ministry of Education-Shanghai Key Laboratory of Children's Environmental Health, Xin Hua Hospital, Shanghai Jiao Tong University School of Medicine, Shanghai, China. ${ }^{3}$ Shanghai Tonxin Pediatric Clinic, Shanghai, China. ${ }^{4}$ Key Laboratory of Dermatology, Ministry of Education, Anhui Medical University, Hefei, Anhui, China. ${ }^{5}$ Department of Pulmonology, Children's Hospital, Zhengzhou University, Zhengzhou, Henan, China. ${ }^{6}$ Department of Pediatrics, Suzhou Wuzhong People's Hospital, Suzhou, Jiangsu, China. ${ }^{7}$ Medical Department, Shanghai Seventh People's Hospital, Shanghai University of Traditional Chinese Medicine, Shanghai, China. ${ }^{8}$ Department of Ophthalmology, Shanghai General Hospital, Shanghai Jiao Tong University School of Medicine, Shanghai, China.

Received: 2 October 2020 Accepted: 28 June 2021

Published online: 09 July 2021

\section{References}

1. O'Connell EJ. The burden of atopy and asthma in children. Allergy. 2004;59(Suppl 78):7-11.

2. Burrows B, Martinez FD, Halonen M, et al. Association of asthma with serum IgE levels and skin-test reactivity to allergens. N Engl J Med. 1989:320:271-7.

3. Hong X, Tsai HJ, Liu X, et al. Does genetic regulation of IgE begin in utero? Evidence from $T(H) 1 / T(H) 2$ gene polymorphisms and cord blood total IgE. J Allergy Clin Immunol. 2010;126(1059-67):1067.e1.

4. Pesonen M, Kallio MJ, Siimes MA, et al. Cord serum immunoglobulin E as a risk factor for allergic symptoms and sensitization in children and young adults. Pediatr Allergy Immunol. 2009;20:12-8.

5. Chen $\mathrm{CH}$, Lee YL, Wu MH, et al. Sex-moderated interactions between IL4/ IL13 pathway genes and prenatal environment on cord blood lgE levels. Clin Exp Allergy. 2019:49:1128-38.

6. Yang KD, Chang JC, Chuang H, et al. Gene-gene and gene-environment interactions on lgE production in prenatal stage. Allergy. 2010;65:731-9.

7. Chen $\mathrm{CH}$, Lee YL, Wu MH, et al. Environmental tobacco smoke and male sex modify the influence of IL-13 genetic variants on cord blood lgE levels. Pediatr Allergy Immunol. 2012;23:456-63.

8. Jacobsen HP, Herskind AM, Nielsen BW, et al. IgE in unselected like-sexed monozygotic and dizygotic twins at birth and at 6 to 9 years of age: high but dissimilar genetic influence on IgE levels. J Allergy Clin Immunol. 2001;107:659-63.

9. Ober C, Hoffjan S. Asthma genetics 2006: the long and winding road to gene discovery. Genes Immun. 2006;7:95-100.

10. Liu X, Beaty TH, Deindl P, et al. Associations between specific serum lgE response and 6 variants within the genes IL4, IL 13 , and IL $4 R A$ in German children: the German Multicenter Atopy Study. J Allergy Clin Immunol. 2004;113:489-95

11. Hizawa N, Yamaguchi E, Jinushi E, et al. A common FCER1B gene promoter polymorphism influences total serum lgE levels in a Japanese population. Am J Respir Crit Care Med. 2000;161:906-9.

12. Woszczek G, Borowiec M, Ptasinska A, et al. Beta2-ADR haplotypes/ polymorphisms associate with bronchodilator response and total lgE in grass allergy. Allergy. 2005;60:1412-7.

13. Hua $L$, Zuo XB, Bao YX, et al. Four-locus gene interaction between IL13, IL4, FCER1B, and ADRB2 for asthma in Chinese Han children. Pediatr Pulmonol. 2016;51:364-71.

14. Sharma V, Michel S, Gaertner $V$, et al. A role of FCER1A and FCER2 polymorphisms in IgE regulation. Allergy. 2014;69:231-6.

15. Chen GB, Liu N, Klimentidis YC, et al. A unified GMDR method for detecting gene-gene interactions in family and unrelated samples with application to nicotine dependence. Hum Genet. 2014;133:139-50.

16. Kelly-Welch AE, Hanson EM, Boothby MR, et al. Interleukin-4 and interleukin-13 signaling connections maps. Science. 2003;300:1527-8. 
17. Pongratz G, McAlees JW, Conrad DH, et al. The level of IgE produced by a $B$ cell is regulated by norepinephrine in a p38 MAPK- and CD23dependent manner. J Immunol. 2006;177:2926-38.

18. Padro CJ, Shawler TM, Gormley MG, et al. Adrenergic regulation of IgE involves modulation of CD23 and ADAM10 expression on exosomes. Immunol. 2013;191:5383-97.

19. Arima K, Umeshita-Suyama R, Sakata Y, et al. Upregulation of IL-13 concentration in vivo by the IL13 variant associated with bronchial asthma. J Allergy Clin Immunol. 2002;109:980-7.

20. Cameron L, Webster RB, Strempel JM, et al. Th2 cell-selective enhancement of human IL13 transcription by IL13-1112C>T, a polymorphism associated with allergic inflammation. J Immunol. 2006;177:8633-42.

21. Toumi A, Abida O, Ben Ayed M, et al. Cytokine gene polymorphisms in Tunisian endemic pemphigus foliaceus: a possible role of il-4 variants. Hum Immunol. 2013;74:658-65.

22. Green SA, Turki J, Bejarano P, et al. Influence of beta 2-adrenergic receptor genotypes on signal transduction in human airway smooth muscle cells. Am J Respir Cell Mol Biol. 1995;13:25-33.

23. Peters JL, Cohen S, Staudenmayer J, et al. Prenatal negative life events increases cord blood lgE: interactions with dust mite allergen and maternal atopy. Allergy. 2012;67:545-51.

24. Wang JY, Chen CA, Hou Yl, et al. Longitudinal pattern of multiplexed immunoglobulin E sensitization from prenatal stage to the first year of life. Pediatr Allergy Immunol. 2016;27:620-6.
25. Hinz D, Simon JC, Maier-Simon C, et al. Reduced maternal regulatory T cell numbers and increased $T$ helper type 2 cytokine production are associated with elevated levels of immunoglobulin E in cord blood. Clin Exp Allergy. 2010;40:419-26.

26. Kim BS, Park SM, Uhm TG, et al. Effect of single nucleotide polymorphisms within the interleukin-4 promoter on aspirin intolerance in asthmatics and interleukin-4 promoter activity. Pharmacogenet Genomics. 2010;20:748-58.

27. Nabavi M, Ghorbani $R$, Asadi AM, et al. Factors associated with cord blood IgE levels. Asian Pac J Allergy Immunol. 2013;31:157-62.

28. Yu J, Ahn K, Shin YH, et al. The interaction between prenatal exposure to home renovation and reactive oxygen species genes in cord blood lgE response is modified by maternal atopy. Allergy Asthma Immunol Res. 2016:8:41-8.

29. Pfefferle PI, Sel S, Ege MJ, et al. Cord blood allergen-specific IgE is associated with reduced IFN-gamma production by cord blood cells: the Protection against Allergy-Study in Rural Environments (PASTURE) Study. J Allergy Clin Immunol. 2008;122:711-6.

\section{Publisher's Note}

Springer Nature remains neutral with regard to jurisdictional claims in published maps and institutional affiliations.
Ready to submit your research? Choose BMC and benefit from:

- fast, convenient online submission

- thorough peer review by experienced researchers in your field

- rapid publication on acceptance

- support for research data, including large and complex data types

- gold Open Access which fosters wider collaboration and increased citations

- maximum visibility for your research: over 100M website views per year

At BMC, research is always in progress.

Learn more biomedcentral.com/submissions 